\title{
Los dispositivos tecnológicos cotidianos en libros de texto. Presencia y análisis de las exposiciones
}

\author{
Manuel Fernández-González ${ }^{1}$, Antonio Jesús Torres-Gil ${ }^{2}$ \\ Departamento de Didáctica de las Ciencias Experimentales. Facultad de Ciencias de la Educación. \\ Universidad de Granada.'mfofaber@ugr.es,2ajtg@yahoo.com
}

[Recibido en febrero de 2014, aceptado en mayo de 2014]

En el presente artículo se estudian los dispositivos tecnológicos cotidianos que presentan los libros de texto de $4^{\circ}$ de ESO (Física y Química). En primer lugar se ha determinado cuáles son los mostrados y si es frecuente su presencia. Se señala que entre los manuales suele aparecer un núcleo más o menos fijo de dispositivos y el resto variable, formado sobre todo por aquellos más actuales. Se ha constatado que la frecuencia con que aparece un dispositivo suele depender de su conexión estrecha con un principio o ley y de su uso extendido, reforzado por su carácter innovador. La comparación realizada con manuales de los años 70 ha revelado un incremento del número de referencias, pero la metodología con que se exponen, igual que en la actualidad, sigue una exposición de corte tradicional, muy deductivista y subordinada a la teoría. Por último se ha acometido el análisis de las propias exposiciones, comprobándose que, en la gran mayoría de los casos, responden a un esquema común constituido por tres elementos: presentación y finalidad, fundamento, y funcionamiento. La ausencia de alguno de ellos conlleva una pérdida de calidad explicativa. El proceso de análisis se ha apoyado en la realización de mapas conceptuales para marcar nítidamente los bloques correspondientes a dichos elementos.

Palabras clave: Dispositivos tecnológicos cotidianos; Ciencia cotidiana; Análisis de libros de texto; Enseñanza contextual.

\section{Everyday technological devices in textbooks: presence and analysis of descriptions}

This article studies the everyday technological devices described in tenth-grade secondary school textbooks. The first step was to determine which devices were included in these books and how frequently they appeared. It was found that there is a more or less fixed nucleus of devices along with other more modern devices, which can vary. The frequency with which a device appears in the textbook depends on the closeness of its connection to a principle or law. It also depends on the wideness of its use, which is reinforced by its innovative nature. The comparison of these textbooks with those used in the 1970s showed an increase in the number of references. However, the methodology, which remained the same as in the past, resulted in explanations that were traditional, deductive, and subordinated to theory. Finally, the textual descriptions of the devices were analyzed. In the majority of cases, these descriptions had the same schema, which was composed of three elements: (i) presentation and purpose; (ii) foundation; (iii) operation. The absence of one of these elements impoverished the quality of the explanation. Conceptual maps were used as an instrument in this analysis to clearly mark the text blocks corresponding to these elements.

Keywords: Everyday technological devices, Everyday science, Analysis of textbooks, Contextual teaching.

\section{Introducción}

Los avances científicos y tecnológicos se hacen cada vez más patentes en la sociedad actual. Las ciencias y la tecnología progresan exponencialmente y los nuevos conocimientos se incorporan a la vida cotidiana materializados en numerosos objetos tecnológicos. Muchos de los dispositivos que utilizamos, aunque muy familiares, encierran unos fundamentos científicos y un funcionamiento que habitualmente se ignoran. Constituyen, pues, auténticas cajas negras para el usuario.

Pero las nuevas orientaciones de la enseñanza de las ciencias prestan una atención creciente a este tipo de contenidos. Desde principios de los años 90 se comienza a señalar la necesidad de promover una "alfabetización científica" (AAAS, 1993), con un currículum más apartado de lo 
académico y más centrado en cuestiones relacionadas con la ciencia y la tecnología del entorno próximo o global (Marco, 2000). Es cierto que las relaciones ciencia escolar - vida cotidiana son controvertidas y por ello han sido objeto de debate, incrementado en los últimos tiempos (Cajas, 1999). La inclusión de objetivos relacionados con esta temática en los curricula de países avanzados responde a las nuevas expectativas de la educación, entre las que se encuentra el conseguir para la población escolar una alfabetización científico-tecnológica de nivel adecuado (Jenkins, 2010).

Dentro del citado enfoque está presente la enseñanza de la ciencia en contexto, orientación didáctica que ha adquirido hoy día una singular importancia (Pilot y Bulte, 2006). Su puesta en práctica supone una apertura al mundo real y rompe con el esquema tradicional de ciencia centrada en sí misma, lo que lleva consigo una disminución de los excesos teóricos de la enseñanza practicada habitualmente (Millar y Hunt, 2002). La ciencia contextual produce, en general, un incremento de la motivación y unos resultados de aprendizaje comparables, al menos, a los de la enseñanza habitual (Bennett et al., 2007).

Entre los contextos que se señalan (De Jong, 2006), el que se refiere al dominio personal o cotidiano es, bajo el punto de vista escolar, el más importante. La ciencia cotidiana presta atención especial al estudio de fenómenos, aparatos y materiales de nuestra vida diaria (Costa, 1995). Se pretende así que los alumnos puedan entender e interaccionar con los avances de nuestros tiempos, estudiando el funcionamiento de aparatos y dispositivos que se usan de forma ordinaria (Olartecoechea, 2003), y las implicaciones derivadas de su uso (Solbes y Vilchez, 1997). Lo cotidiano no sólo motiva sino que también permite una enseñanza más sugestiva y más cercana a los intereses de los alumnos, sin que esto conlleve necesariamente pérdida de rigor (Aragón, 2004).

La orientación ciencia cotidiana puede entenderse de varias maneras. En su sentido más innovador, como una propuesta de enseñanza que estructura un curso de ciencias en torno a lo cotidiano. Tendencia en auge, pero por el momento minoritaria. Un ejemplo prototípico es el Proyecto Salters (Campbell et al., 1994; Plana et al., 2005). Otra alternativa, que viene de tiempo atrás y que se sitúa al margen de propuestas metodológicas, consiste en introducir ocasionalmente en el programa tradicional alguna parcela de lo cotidiano (Jiménez-Liso y De Manuel, 2009).

Todos estos movimientos surgidos en el seno del ámbito educativo son parte de la respuesta a la situación de crisis que desde hace varias décadas vive la enseñanza de las ciencias, manifestada por el rechazo a su estudio de muchos alumnos. Aunque las causas son complejas, no cabe duda que gran parte de responsabilidad debe atribuirse al planteamiento que mantiene su enseñanza (Fernández, 2008), la cual sigue mostrando: 1) una imagen de ciencia centrada en sí misma, académica y formalista; 2) una falta de conexión con la ciencia presente en el mundo cotidiano o los medios de comunicación; 3) una ciencia que no atiende a aspectos de su propia naturaleza. Las instituciones oficiales han tomado ya conciencia de esta preocupante situación y de sus consecuencias. Así, la Comisión Europea, en un reciente informe (Rocard et al., 2008) ha alertado sobre el "peligro capital para el futuro de Europa" que supone el despoblamiento de las carreras de ciencias.

Nuestro ordenamiento educativo, como sabemos, ha incorporado a nuestros planes de estudio una asignatura obligatoria, Ciencias para el Mundo Contemporáneo, de orientación "ciencia para todos". Igualmente, y siguiendo a los países más avanzados, proclama la necesidad de incorporar a la enseñanza contenidos del ámbito tecnológico. El Decreto de enseñanzas mínimas de la ESO lo expresa en uno de los objetivos del área de Ciencias de la Naturaleza: Comprender y utilizar los conceptos básicos de las ciencias de la naturaleza para interpretar los fenómenos 
naturales, asi como para analizar y valorar las repercusiones de desarrollos tecnocientificos y sus aplicaciones (M.E.C., 2007: 693).

En esta investigación nos proponemos estudiar la presencia en el medio escolar de los dispositivos tecnológicos cotidianos a través de la información que nos ofrecen los libros de texto. Concretamente, nos hemos situado en Secundaria, a nivel de $4^{\circ}$ de ESO (Física y Química). Pretendemos determinar qué dispositivos presentan, de qué manera lo hacen y cuál es el papel que se les asigna. Igualmente pasaremos a comprobar si hay signos de evolución en relación a libros de texto menos actuales. Nos hemos detenido por último en investigar qué elementos estructuran las exposiciones, para comprobar si responden a un esquema común que posteriormente pueda guiarnos en el análisis de cualquiera de ellas.

\section{¿Qué entendemos por dispositivo tecnológico cotidiano?}

Puesto que el objeto de estudio de nuestro trabajo son los dispositivos tecnológicos cotidianos, es obligado comenzar aclarando el término, especialmente los matices derivados de los adjetivos "tecnológico" y "cotidiano".

En una primera aproximación, por dispositivo tecnológico puede entenderse un objeto o sistema que aúna ciencia y tecnología, y es utilizado por el hombre para mejorar su calidad de vida y el funcionamiento de la sociedad en que vive. Por lo que respecta a la parte material, el dispositivo consta de un mecanismo formado por diversos elementos que actúan conjuntamente para obtener un resultado automático. Así pues, sus rasgos esenciales son: la base tecnológica de su diseño, la diversidad de componentes, la existencia de un mecanismo de funcionamiento (normalmente no evidente) y la actuación conjunta hacia un fin prefijado.

Por lo que acaba de decirse van a ser excluidos de nuestro estudio, aunque aparezcan en los libros de texto, instrumentos sin mecanismo, (p.ej. el plano inclinado), o instrumentos artísticos (p.ej. piano), que pertenecen al ámbito artesanal más que al tecnológico (otro caso sería algún instrumento como la guitarra eléctrica).

En cuanto a lo "cotidiano" es entendido como un término que engloba todos aquellos objetos, fenómenos y situaciones con los que nos relacionamos frecuentemente en nuestras actividades diarias (Morales y Manrique, 2012). Si atendemos entonces al significado así expresado, hemos de descartar tanto a los aparatos históricos (p.ej. la marmita de Papin), como a aquellos de ultimísima generación (y de porvenir aún incierto), introducidos con la sola finalidad de hacer notar la puesta al día del manual.

En el mismo sentido y para concretar más el término, vamos a distinguir entre dispositivos e instrumentos científicos (p.ej. osciloscopio). La diferencia es que los dispositivos tienen una utilidad cotidiana y una incidencia social muy amplia. Los instrumentos científicos, entre los que se encuentran los instrumentos de medida con escalas cuantitativas (p.ej. amperímetro), son de uso minoritario, aunque alguno de ellos pueda estar en casa (p.ej. barómetro, termómetro).

Entre la generalidad de dispositivos, los dispositivos cotidianos son los que pertenecen a nuestro entorno experiencial. Sobre ellos solemos tener experiencia real y directa (p.ej. frigorífico, teléfono móvil), o experiencia virtual a través de la TV (p.ej. aerodeslizador). Los hay de utilización individual y de utilización colectiva; pueden estar en casa, fuera de ella, y en los medios de comunicación.

Aunque hemos restringido el campo de "dispositivo", no por ello ha perdido variedad. Lo mismo puede incluir aparatos habituales en el hogar (p.ej. frigorífico), que instrumentos que forman parte de otros más complejos (p.ej. freno hidráulico en automóviles), máquinas 
destinadas al transporte (p.ej. tren de levitación magnética), aparatos recreativos (p.ej. juguete de cuerda), etc. Bajo el punto de vista didáctico, los dispositivos cotidianos constituyen un grupo muy interesante, pues permiten al profesor utilizarlos para fines disciplinares y, al mismo tiempo, mostrar al alumno que vive en un mundo repleto de ciencia.

\section{Metodología}

En nuestra investigación hemos comenzado estudiando la presencia global de los dispositivos en los manuales y siguiendo una línea de acercamiento progresivo al dispositivo mismo.

\section{A. Presencia de los dispositivos}

A1. En primer lugar se pretende valorar la presencia de los dispositivos tecnológicos cotidianos en manuales de $4^{\circ}$ de ESO (Física y Química). Una mayor presencia suele ir asociada a un mayor interés por conectar con el mundo vivencial del alumno. Este estudio va a llevarse a cabo sobre una muestra constituida por 10 libros de texto de editoriales de amplia difusión (ver Anexo 1). Se procede entonces a la revisión de los manuales a fin de localizar los dispositivos que muestran, teniendo en cuenta las precisiones señaladas en el apartado anterior. La simple mención, por supuesto, no se considera. Se determina así cuáles son, si es frecuente su presencia, y la razón de la misma.

A2. Hemos creído también interesante realizar un estudio comparativo utilizando manuales de $2^{\circ}$ de BUP de los años 70, que puede proporcionar datos acerca de la posible evolución experimentada por los dispositivos y sus exposiciones hasta la actualidad. Tomamos entonces 6 libros de $2^{\circ}$ de BUP, curso correspondiente al actual $4^{\circ}$ de ESO, y se contrastaron con otros 6 libros de este último, de entre los 10 anteriores (Anexo 2). Para salvar el obstáculo de la diferencia de los programas hemos considerado únicamente los temas que comparten (cinemática, dinámica, hidrostática, energía, sonido y calor).

\section{B. Relevancia de los dispositivos}

Una vez cumplida esta etapa, se pasa a determinar en cada referencia la mayor o menor atención prestada al dispositivo por el manual y el papel que se le asigna. Un primer contacto con las exposiciones apunta a la existencia de dos modalidades principales:

Tipo-a. El dispositivo con cierto nivel de relevancia

Unas veces se manifiesta por el contexto que la finalidad de la presentación es, sobre todo, el estudio del dispositivo por sí mismo. Como consecuencia, manteniendo la vinculación a su base teórica, se presta una especial atención al funcionamiento, que gana peso en la exposición.

Aparte de su mayor extensión, una prueba inequívoca de esta modalidad es que el título bajo el que aparece está referido al nombre del dispositivo y no a conceptos o fenómenos disciplinares.

En esta modalidad se le concede al dispositivo la máxima relevancia frente a la teoría. Y puede incluso aportar elementos teóricos nuevos. La modalidad deja traslucir la influencia del enfoque ciencia contextual.

Tipo- $b$. El dispositivo como puro ejemplo de la teoría

El dispositivo figura subordinado totalmente a la teoría, como puro ejemplo de las ideas que constituyen su fundamento. Aparece bajo el título del concepto o ley que ejemplifica. La intencionalidad suele ser mostrar que la teoría está presente en el mundo real, sin aportar 
muchos detalles del ámbito interno del dispositivo. Por ello su presencia suele ser muy escueta.

Pero esta modalidad de presentación puede encerrar, bien diseñada, un interés didáctico especial porque en pocas líneas ofrece la información esencial del dispositivo.

Pretendemos entonces comprobar estas tipologías (a y b) en una muestra extensa de exposiciones.

\section{Análisis de las exposiciones}

Se pasa entonces a realizar un análisis de las exposiciones individuales. El objetivo es indagar si presentan una estructura semejante con elementos comunes. Buscamos especialmente tres elementos constituyentes: presentación y finalidad, fundamento, y funcionamiento, ya que dan respuesta a las preguntas esenciales sobre el dispositivo: ¿qué es y para qué sirve? ¿por qué funciona? y ¿cómo funciona? Sus características se indican a continuación.

Presentación (¿Qué es?). Da idea de qué es el aparato y describe de modo abreviado lo más fundamental del mismo. Es imprescindible para un dispositivo poco habitual (p.ej. el aerodeslizador) y debería ir complementada por una ilustración. Pero en caso de aparatos muy conocidos, la presentación puede obviarse.

Dentro de la presentación hemos incluido la finalidad (¿para qué sirve?), ya que forma parte de la descripción del dispositivo. Indica la misión del mismo, es decir, el fin práctico que produce al actuar. Ha de verse bajo el punto de vista del usuario (¿para qué lo compra?). Por ejemplo, la finalidad del micrófono es recoger el sonido.

Fundamento (¿Por qué funciona?). Es el elemento teórico que explica el funcionamiento del aparato. Puede residir en alguna característica del agente que lo hace funcionar (p.ej. la fuerza expansiva del vapor), en un fenómeno (p.ej. el efecto fotoeléctrico), o en un principio (p.ej. la transformación de la energía). Tiene carácter general ya que puede ser el sostén teórico de muchos y diferentes aparatos, fenómenos o procesos. Habitualmente el fundamento de un dispositivo viene dado por la teoría estudiada en el mismo apartado que lo incluye.

Funcionamiento (¿Cómo funciona?). Justifica, con base en el fundamento, la actuación del aparato. Se establece una conexión de lo teórico con lo real, al ser aplicada la teoría (el fundamento) a un caso particular (el dispositivo y su funcionamiento). Es evidente, además, que en la mayor parte de los casos, para explicar el funcionamiento del aparato, se hace necesario reseñar los componentes y su actuación conjunta, es decir, el mecanismo del aparato.

Comenzaremos entonces esta etapa analizando sobre una muestra reducida pero variada (Anexo 3) si están presentes los tres elementos fundamentales. A continuación se procede a comprobar si el resto de las exposiciones están constituidas por dichos elementos. El proceso de análisis, apoyado en la realización de mapas conceptuales (Starr y Krajcik, 1990), va a permitir decidir sobre la idoneidad de la propuesta.

\section{Resultados y discusión}

\section{A1. Presencia de los dispositivos}

Se examinaron los 10 manuales de $4^{\circ}$ de ESO para localizar los dispositivos tecnológicos cotidianos que incluían. Han aparecido 47 dispositivos diferentes, que se muestran en un total de 145 ocasiones. Los más frecuentes, es decir, los que están presentes en cinco o más manuales, se han incluido en la tabla 1. En ella algunos van emparejados (se cuentan como 
uno) ya que muestran el mismo fundamento y casi igual funcionamiento. Obviamente van a faltar dispositivos de los campos no considerados en el programa de la asignatura (p.ej. óptica).

Tabla 1. Dispositivos más frecuentes en 10 libros de texto de $4^{\circ}$ de ESO.

\begin{tabular}{|c|c|c|}
\hline Dispositivo & $\begin{array}{c}\text { Manuales } \\
\text { que lo muestran }\end{array}$ & $\begin{array}{c}\text { En } \\
\text { tipo-a }\end{array}$ \\
\hline Elevador/Prensa hidráulica & 10 & 10 \\
\hline Motor de combustión interna & 10 & 8 \\
\hline Máquina de vapor & 9 & 5 \\
\hline Globo/Dirigible & 8 & 3 \\
\hline Freno hidráulico & 7 & 4 \\
\hline Barco & 7 & 0 \\
\hline Polea/Polipasto & 6 & 3 \\
\hline Frigorífico/Bomba de calor & 5 & 1 \\
\hline Aerogenerador & 5 & 1 \\
\hline Submarino & 5 & 0 \\
\hline Placa fotovoltaica & 5 & 0 \\
\hline Ecógrafo/Sónar & 5 & \\
\hline
\end{tabular}

Los dispositivos más mencionados y que se exponen con mayor detenimiento (tipo-a) resultan ser los que están ligados estrechamente a una ley o principio teórico. Así, el elevador/prensa hidráulica es un ejemplo prototípico del principio de Pascal; la máquina de vapor, del segundo principio de la termodinámica; el motor de combustión interna, también. Su importancia, además, es incuestionable por ser en gran parte responsables del desarrollo de la sociedad moderna. Otros dispositivos (barco, submarino) son artefactos complejos de los que sólo se estudia una propiedad esencial (la flotabilidad) y otros relativamente recientes (aerogenerador, frigorífico/bomba de calor, placa fotovoltaica, ecógrafo/sonar) afianzan poco a poco su presencia en las páginas de nuestros manuales.

El resto de dispositivos que no figura en la tabla son en su gran mayoría muy de actualidad. Destacan con 3-4 menciones: convertidor catalítico, GPS, horno microondas, láser y pila de combustible. $\mathrm{Y}$ entre los menos citados los hay de fundamento considerado poco asequible (p.ej. airbag), o de uso más minoritario (p.ej. visor de IR).

En resumen, a grandes rasgos, nos encontramos en el conjunto de los dispositivos con un núcleo fijo que se repite en los distintos manuales y el resto variable, constituido por los más modernos. Entre estos, los de más actualidad, aún no consolidados, ocupan un lugar inferior en la escala de presencia. Por otra parte, hay que hacer notar que si el dispositivo es un artefacto complejo suele rehuirse su tratamiento (p.ej. el teléfono móvil, sólo mencionado por 
un manual), a menos que se decida estudiar de él sólo una parte esencial o fenómeno (p.ej. en el barco, la flotabilidad; en la lavadora, la centrifugación).

\section{A2. Evolución en manuales}

Se dan ahora los resultados del contraste de exposiciones de $2^{\circ}$ de BUP y de $4^{\circ}$ de ESO actuales. La tabla 2 ofrece el número de dispositivos diferentes que aparece en ambas épocas y el número total de veces que se citan en el conjunto de cada muestra (van entre paréntesis las citas de tipo-a).

Tabla 2. Número global de dispositivos en 6 manuales antiguos y 6 actuales (temas comunes).

\begin{tabular}{|c|c|c|}
\hline & $\mathbf{2}^{\mathbf{o}} \mathbf{B U P}$ & $\mathbf{4}^{\mathbf{o}} \mathbf{E S O}$ \\
\hline Dispositivos diferentes & 19 & 31 \\
\hline Dispositivos, citas & $35(12)$ & $78(39)$ \\
\hline Media manual & 6 & 13 \\
\hline
\end{tabular}

En la tabla 3 se da, además, un extracto con ejemplos significativos que aparecen en las dos muestras.

Tabla 3. Ejemplos de dispositivos en 6 manuales antiguos y 6 actuales (temas comunes).

\begin{tabular}{|c|c|c|}
\hline Dispositivo & $\mathbf{2}^{\mathbf{o}} \mathbf{B U P}$ & $\mathbf{4}^{\mathbf{}} \mathbf{E S O}$ \\
\hline Aerodeslizador & & $2(2)$ \\
\hline Aerogenerador & & $2(1)$ \\
\hline Barco & 3 & 4 \\
\hline Bomba aspirante & $1(1)$ & \\
\hline Elevador/Prensa hidráulica & $6(4)$ & $6(6)$ \\
\hline Frenos hidráulicos & 5 & $5(2)$ \\
\hline Frigorífico & $1(1)$ & $4(3)$ \\
\hline Globo/Dirigible & 1 & $5(2)$ \\
\hline Horno microondas & & $3(1)$ \\
\hline Láser & $3(2)$ & $2(1)$ \\
\hline Máquina de vapor & $3(3)$ & $6(3)$ \\
\hline Motor de combustión interna & & 2 \\
\hline Placa fotovoltaica & & 1 \\
\hline Teléfono móvil & 1 & \\
\hline Termo & &
\end{tabular}

Los resultados globales de ambas muestras (tabla 2) señalan una mayor atención hacia los dispositivos en nuestros días que en los años 70. Esto viene indicado por la variedad que encontramos: 19 ( $2^{\circ}$ BUP) frente a 31 ( $\left.4^{\circ} \mathrm{ESO}\right)$, así como por el número de veces que se citan: 35 frente a 78, lo que supone una media por manual de 6 frente a 13. En el mismo sentido apunta el número de dispositivos tipo-a que aparecen, que pasa de la tercera parte (12) a la mitad (39). 
En cuanto a dispositivos concretos (tabla 3), encontramos en $2^{\circ}$ de BUP, presentes y con gran relieve, los tradicionales como frenos, elevador hidráulico, barco, motor de combustión, etc. Son aparatos tecnológicos que no han perdido vigencia y muestran una conexión estrecha y directa con importantes leyes y principios. En cambio hay otros en $4^{\circ}$ de ESO que han surgido como consecuencia de la rápida evolución tecnológica experimentada en los últimos tiempos, como aerogenerador, aerodeslizador, horno microondas, láser, placa fotovoltaica, teléfono móvil, etc. También tenemos ejemplos de lo contrario, esto es, dispositivos que han desaparecido como bomba aspirante, termo, etc. Otros, por último, aparecen en los manuales de una época u otra sin más significación que la decisión de los autores.

\section{B. Relevancia de los dispositivos}

En el estudio llevado a cabo es de señalar que, en la secuencia expositiva, el dispositivo se sitúa después de la teoría. Las exposiciones suelen seguir, pues, una línea de corte tradicional. Nunca aparece como objeto en el que reside un concepto estructurante que sirve de punto de partida al desarrollo de una temática. Tampoco hemos encontrado exposiciones que sigan otras metodologías inductivas, como investigación dirigida o resolución de problemas.

Sin embargo, en la línea indicada se ha constatado que las exposiciones encajan fácilmente en una de las dos modalidades señaladas, tipo-a o tipo-b, según el mayor o menor protagonismo concedido al dispositivo y, consecuentemente, las finalidades perseguidas.

Las de tipo-a vienen con frecuencia ubicadas en un recuadro o sección fija complementaria de la unidad, aunque pueden también aparecer en el núcleo de la misma, ocupando un apartado propio (p.ej. motor de combustión interna). En cuanto a las de tipo-b, son las que suelen predominar en buena parte de los manuales. Su desarrollo es normalmente muy reducido y rara vez pasan de un párrafo.

Pero esta modalidad-b puede encerrar, bien diseñada, un interés didáctico especial porque en pocas líneas ofrece la información esencial del dispositivo. Por ejemplo, sobre la televisión algún manual dice "Es un aparato complejo que transforma el sonido y las imágenes en OEM de frecuencias intermedias que pueden ser enviadas a grandes distancias" (Anaya, p.125).

En cambio, puede también ocurrir que en la modalidad-b falte una información tan esencial como el funcionamiento. Por ejemplo, al hablar del hidrógeno como combustible se dice escuetamente: "El hidrógeno se utiliza en unos dispositivos denominados pilas de combustión que se utilizan como fuente de energía en la industria aeroespacial y, más recientemente, en algunos automóviles" (Santillana, p.248). No obstante, también debe señalarse que una descripción tipo-a no garantiza la calidad de lo expuesto, que a veces puede mostrarse francamente escasa (p.ej. la cámara termográfica, Bruño, p.211).

\section{Análisis de las exposiciones}

El objetivo propuesto era perfilar los elementos componentes de la exposición. Revisadas entonces las exposiciones de la muestra reducida (Anexo 3) se observa que el esquema anterior, constituido por los tres elementos fundamentales, está bien presente en ellas. En las de tipo-b de modo menos nítido, debido a su abreviado desarrollo, siendo frecuentes los solapamientos y, a veces, las omisiones. Por lo cual la tarea de análisis se hace más ardua. De todos modos se ha contado con la ayuda de mapas conceptuales, que resaltan los bloques estructurales de la exposición (Pontes, 2012).

La idoneidad del esquema se confirma al repetir el análisis en una muestra más amplia (42 exp., Anexo 4), si bien no es raro que alguno de los tres elementos falte o esté implícito, lo que es muy común en exposiciones tipo-b. Lo que sí es frecuente en ambas modalidades, es que 
los elementos no siempre aparecen bien perfilados ni siguiendo un orden lógico: unas veces están separados y otras entremezclados, unas secuenciados de una manera y otras, de otra diferente. De cualquier modo, la presencia de todos en la exposición es un indicio positivo pero no suficiente, pues ésta puede resultar inadecuada por defecto grave de alguno de ellos.

Es interesante también señalar que algunas veces aparece algún elemento adicional como, por ejemplo, señalamientos históricos, alusiones medioambientales, o vínculos con otros aparatos semejantes. Pero se prodigan raramente (ninguno llega al 10\%) y, además, podrían ser asumidos fácilmente en alguno de los tres elementos fundamentales.

\section{Análisis de exposiciones. Ejemplos}

A fin de ilustrar el análisis de las exposiciones llevado a cabo, y que acaba de ser referido en el apartado anterior, ofrecemos aquí dos ejemplos concretos: el primero acerca de una exposición tipo-a y el segundo de otra tipo-b. En ambos casos se han dibujado los mapas conceptuales, que consiguen resaltar, mejor que el texto, varios bloques que se corresponden con los elementos fundamentales. Obsérvese que el bloque "funcionamiento" se encuentra conectado al bloque "fundamento". Esto ha de ocurrir siempre que el planteamiento sea correcto.

\section{Aerodeslizador (SM 4 ${ }^{\circ}, 2008$, p.56)}

La información ofrecida en el manual consta de cuatro párrafos (en el recuadro se han marcado con números), acompañados de una fotografía del aparato.

[1] "Un aerodeslizador es un vehiculo que se sostiene sobre una superficie lisa, sin tener contacto con ella, gracias a un chorro de aire que lanza contra la superficie. Entre el vebiculo y la superficie se crea un colchón de aire que le permite moverse sobre superficies lisas, como el agua, pudiendo alcanzar velocidades de $150 \mathrm{~km} / \mathrm{h}$. Sobre tierra puede llegar a $400 \mathrm{~km} / \mathrm{h}$.

[2] La fuerza que ejerce el chorro de aire contra la superficie es respondida por esta, de acuerdo al tercer principio de la dinámica, realizando una fuerza hacia arriba que sostiene el peso del aerodeslizador separándolo de la superficie.

[3] El primer aerodeslizador de pasajeros que realizó viajes regularmente en las costas de W ales lo bizo en 1961. Se sostenia por turbopropulsores que lanzaban el aire contra la superficie sobre la que se deslizaba, avanzaba mediante bélices y era conducido con un timón.

[4] El mismo sistema de propulsión que utiliza el aerodeslizador para sostenerse sin entrar en contacto con la superficie sobre la que se desliza es la base de los motores de reacción o de propulsión a chorro, con la que se mueven este tipo de aviones. Cuando los gases de escape se lanzan hacia atrás, el avión es impulsado bacia delante aumentando su velocidad."

Comentemos ahora la presencia de los elementos constituyentes en los distintos párrafos.

[1] Corresponde esencialmente a una presentación del dispositivo, caracterizada por el resumen abreviado de lo más típico del mismo. Puesto que es poco conocido, procede añadir una fotografía y comenzar aclarando que se trata de un vehículo que se mueve sobre el agua. Se aprecia también un avance del funcionamiento y la finalidad. El primero, "se sostiene...gracias a un chorro de aire que lanza contra la superficie", se repetirá en [3]. En cuanto a la finalidad, se cita aquí y se completa en [3]: es un vehículo que sirve para el transporte de pasajeros por mar a gran velocidad. 
[2] Muestra sin ambigüedad el fundamento teórico ("de acuerdo al tercer principio de la dinámica"), que sirve de soporte explicativo al funcionamiento: "La fuerza que ejerce el chorro de aire contra la superficie es respondida... realizando una fuerza hacia arriba que sostiene el peso del aerodeslizador".

[3] Vuelve a repetir el mecanismo de sustentación y completa el funcionamiento, señalando la causa del desplazamiento horizontal ("avanzaba mediante hélices").

[4] Ofrece un vínculo contextual de interés con los motores de reacción de los aviones, señalando que ambos poseen un fundamento y funcionamiento semejante.

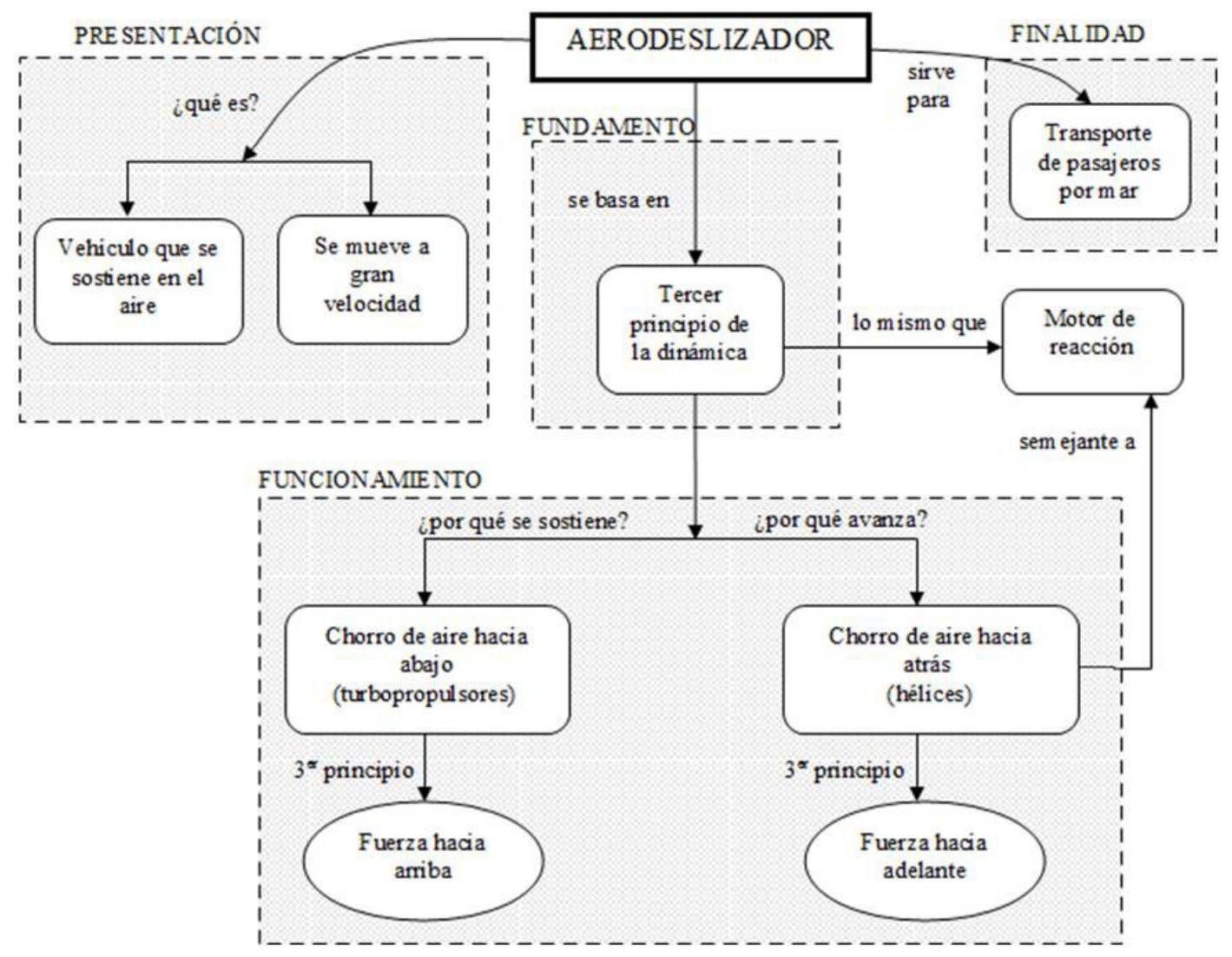

Panel fotovoltaico (SM 4 ${ }^{\circ}, 2008$, p.120)

Aunque ofrece una información mínima sobre el dispositivo, esta recoge lo más esencial del mismo. Va acompañada de una fotografía. El ejemplo, dentro de lo escueto, puede considerarse aceptable.

"Fuentes renovables de energía. El Sol."

"La energía solar fotovoltaica aprovecha el efecto fotovoltaico: cuando la luz incide sobre un material semiconductor se genera un flujo de electrones. Las células fotovoltaicas convierten la energía solar en energía eléctrica".

La exposición se encuentra desordenada y revuelta, por lo que el análisis no es tan fácil como el anterior. El título ("Fuentes renovables de energía. El Sol”) orienta acerca de la finalidad del dispositivo, que es recoger la energía solar. Lo poco que se dice del dispositivo es insuficiente para hacerse una idea de él y la fotografía que lo acompaña no contribuye mucho (¿cómo es? ¿quién lo utiliza? ¿dónde se pone?). Lo único que puede entresacarse con relación a la presentación es que el panel está hecho de material semiconductor. 
Encontramos el fundamento cuando se señala al "ffecto fotovoltaico" como responsable del proceso en que "las células fotovoltaicas convierten la energía solar en energía eléctrica". En cuanto al funcionamiento sólo se menciona lo esencial: "cuando la luz incide sobre un material semiconductor [el panel] se genera un flujo de electrones". No se dan ni más detalles, ni esquema alguno.

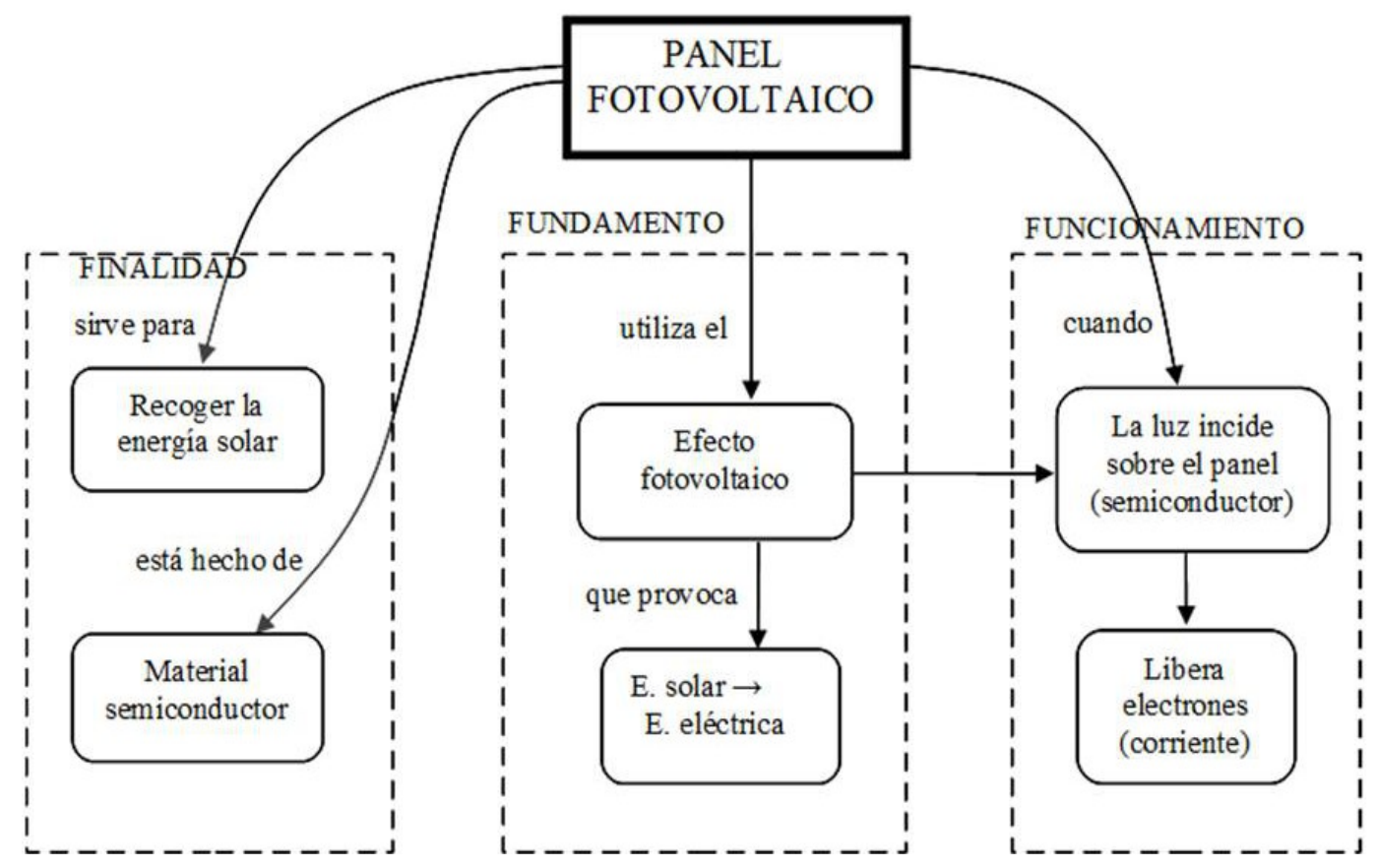

\section{Conclusiones}

En el presente trabajo se ha realizado un estudio sobre los dispositivos tecnológicos cotidianos en manuales de $4^{\circ}$ de ESO. Se ha comprobado que, en general, la presencia es razonable, aunque nada excesiva. Sobre su distribución en los distintos manuales, suele aparecer un núcleo fijo de dispositivos (tabla 1) y el resto variable, formado sobre todo por los más actuales. Entre las exposiciones mostradas, la mitad (tabla 2) está centrada en el dispositivo (tipo-a), con especial atención al funcionamiento del mismo. La otra mitad ofrece una información muy reducida (tipo-b) y se emplea en especial para ejemplificar la teoría. En todo caso, las exposiciones suelen seguir una metodología de corte tradicional, con secuencias deductivas y siempre subordinadas a la teoría. Constituyen, pues, ejemplos de contenidos actuales, expuestos de manera tradicional.

Comparando nuestros manuales de hoy con los de los años 70 , hemos constatado un incremento del número de referencias y, por tanto, un mayor interés hacia lo contextual (tabla 2). En cuanto a la metodología de exposición, no se diferencia gran cosa de la actual y se encuentra igualmente al servicio de la teoría. Es destacable el caso de ciertos dispositivos (p.ej. el elevador hidráulico) que constituyen una materialización de la teoría y por ello reciben una atención preferente de los manuales de antes y de ahora. Otro rasgo a señalar es que el colectivo de aparatos va cambiando con el tiempo, acogiendo nuevos dispositivos y eliminando otros por obsoletos (tabla 3).

En el estudio de la presencia de los dispositivos se ha puesto de manifiesto que la frecuencia con que aparecen está relacionada con dos factores. Uno es su conexión estrecha con un principio o ley (p.ej. el elevador con el principio de Pascal). Otro es el uso extensivo que la sociedad hace de él, reforzado, si es el caso, por su carácter innovador (p.ej. las placas 
fotovoltaicas). El primero justifica la permanencia en los textos de algunos dispositivos tradicionales; el segundo avala la incorporación de los nuevos.

Se ha acometido, también, el análisis de las exposiciones individuales, comprobándose que suelen estar constituidas por tres elementos fundamentales: presentación y finalidad, fundamento, y funcionamiento, este último en conexión con el fundamento. La omisión de alguno, que a veces sucede, conlleva una pérdida evidente de calidad explicativa. A este esquema responden tanto las exposiciones tipo-a como las de tipo-b, lo que puede hacerse muy evidente a través de mapas conceptuales.

El esquema propuesto puede servir como guía de análisis para determinar la calidad de las exposiciones de un manual, así como para elaborar, al margen del manual, otras sobre dispositivos de marcado interés para los alumnos por su proximidad y uso.

\section{Referencias bibliográficas}

AAAS (American Association for the Advancement of Science) (1993). Benchmarks for Science Literacy. New York: Oxford University Press.

ARAGÓN MÉNDEZ, M.M. (2004). La ciencia de lo cotidiano. Revista Eureka sobre Enseñanz̧a y Divulgación de la Ciencia, 1(2), 109-121.

BENNETT, J., LUBBEN, F. y HOGARTH, S. (2007). Bringing Science to Life: A Synthesis of the Research Evidence on the Effects of Context-Based and STS Approaches to Science Teaching. Science Education, 91(3), 347-370.

CAJAS, F. (1999). Public understanding of science: using technology to enhance school science in everyday life. International Journal of Science Education, 21(7), 765-773.

CAMPBELL, B., LAZONBY, J., MILLAR, R., NICOLSON, P., RAMSDEN, J. y WADDINGTON, D. (1994). Science: The Salters' approach. A case study of the process of large scale curriculum development. Science Education, 78(5), 415-447.

COSTA, V. (1995). When science is "another world": Relationships between worlds of family, friends, school, and science. Science Education, 79(3), 347-372.

DE JONG, O. (2006). Making chemistry meaningful: Conditions for sucessful context-based teaching. Educación Quimica, 17(2), 215-221.

FERNÁNDEZ-GONZÁLEZ, M. (2008). Ciencias para el mundo contemporáneo. Algunas reflexiones didácticas. Revista Eureka sobre Enseñanza y Divulgación de las Ciencias, 5(2), 185-199.

JENKINS, E. (2010). How might research inform scientific literacy in schools? Education in Science, 239, 26-27.

JIMÉNEZ-LISO, M.R. y DE MANUEL, E. (2009). El regreso de la química cotidiana: ¿Regresión o innovación? Enseñanz̧a de las Ciencias, 27(2), 257-272.

MARCO-STIEFEL, B. (2000). La alfabetización científica. En Perales y Cañal (Dirs.) Didáctica de las Ciencias Experimentales, pp. 141-164. Alcoy: Marfil.

MILLAR, R. y HUNT, A. (2002). Science for public understanding: a different way to teach and learn science. School Science Review, 83(304), 35-42.

MINISTERIO DE EDUCACIÓN Y CIENCIA (2007). Real Decreto 1631/2006 por el que se establecen las enseñanzas mínimas correspondientes a la Educación Secundaria Obligatoria. B.O.E., 5, 05-01-2007. Madrid: M.E.C. 
MORALES PÉREZ, R.W. y MANRIQUE RODRIGUEZ, F.A. (2012). Formación de profesores de química a partir de la explicación de fenómenos cotidianos: una propuesta con resultados. Revista Eureka sobre Enseñanza y Divulgación de la Ciencia, 9(1), 124-142.

OLARTECOECHEA, C. (2003). Tecnología y Ciencia: Interdisciplinariedad y vinculación con el mundo de las ocupaciones. Alambique 38, pp. 42-48.

PILOT, A. y BULTE, A.M.W. (2006). Why Do You "Need to Know"? Context-based education. International Journal of Science Education, 28(9), 953-956.

PONTES PEDRAJAS, A. (2012). Representación y comunicación del conocimiento con mapas conceptuales en la formación del profesorado de ciencia y tecnología. Revista Eureka sobre Enseñanza y Divulgación de la Ciencia, 9(1), 106-123.

PLANA, O., CAAMAÑO, A., ENRECH, M., PONT, J. y PUEYO, L. (2005). La Física Salters: un proyecto para la enseñanza contextualizada de la física en el bachillerato. Alambique, 46, 93-102.

ROCARD, M., CSERMELY, P., JORDE, D., LENZEN, D., WALWERG-HENRIKSSON, H. y HEMMO, V. (2008). Enseñanza de las ciencias ahora: Una nueva pedagogía para el futuro de Europa. Alambique, 55, 104-117.

SOLBES, J. y VILCHES, A. (1997). STS Interaction and the Teaching of Physics and Chemistry. Science Education, 81, 377-386.

STARR, M.L. y KRAJCIK, J.S. (1990). Concept maps as a heuristic for science curriculum development: Toward improvement in process and product. Journal of Research in Science Teaching, 27(10), 987-1000.

\section{Anexo}

1. Libros de $4^{\mathbf{o}}$ de ESO revisados (Se da la editorial. Todos ellos publicados en 2008): Anaya, Bruño, Edelvives., Editex, Everest, McGrawHill, Oxford, Santillana, SM y Vicens Vives.

2. Contrastación libros $\mathbf{2}^{\circ} \mathbf{B U P}-4^{\circ} \mathbf{E S O}$ (los primeros son de los años 1970s, los segundos de 2008):

Anaya, Bruño, Everest, Santillana, SM y Vicens Vives (La editorial es la misma en ambos casos).

3. Análisis de exposiciones. Muestra inicial (Tomada de manuales de $4^{\circ}$ de ESO, 2008)

- Tipo-a: Aerodeslizador, SM, p.56 // Cámara termográfica, Bruño, p.211 // Acumulador, Bruño, p.51.

- Tipo-b: Micrófono, Anaya, p.124 // Pila de combustible, Santillana, p.248 // Panel fotovoltaico, SM, p.120.

4. Análisis de exposiciones. Muestra amplia (tomada de los tres primeros libros de $4^{\circ}$ de ESO. Ver A1).

Anaya: 16 (5+11); Bruño: 16 (10+6); Edelvives: 10 (5+5). Total: 42 exposiciones (20 tipo-a +22 tipo-b). 